\title{
Formación educativa en y desde las Tecnologías de Información y Comunicación (TIC) en educación secundaria: el reto de hoy
}

Mercedes Estrada, José; Bennasar García, Miguel Israel

Formación educativa en y desde las Tecnologías de Información y Comunicación (TIC) en educación secundaria: el reto de hoy

Revista Educación, vol. 45, núm. 2, 2021

Universidad de Costa Rica, Costa Rica

Disponible en: https://www.redalyc.org/articulo.oa?id=44066178017

DOI: https://doi.org/10.15517/revedu.v45i1.43424

\section{(c) $($ ) $\Theta$}

Esta obra está bajo una Licencia Creative Commons Atribución-NoComercial-SinDerivar 3.0 Internacional. 
Artículos científicos de experiencia social o didáctica

\title{
Formación educativa en y desde las Tecnologías de Información y Comunicación (TIC) en educación secundaria: el reto de hoy
}

\author{
Information and Communication Technologies (ICTs) in Secondary School Education: A Contemporary \\ Challenge
}

José Mercedes Estrada

Universidad Pedagógica Experimental Libertador,

Venezuela

DOI: https://doi.org/10.15517/revedu.v45i1.43424

Redalyc: https://www.redalyc.org/articulo.oa?

estradajme@hotmail.com

iD https://orcid.org/0000-0003-2542-9260

Miguel Israel Bennasar Garcia

Instituto Superior de Formación Docente Salomé Ureña,

República Dominicana

miguelbennasar7884@gmail.com

iD https://orcid.org/0000-0002-3856-0279

Recepción: 20 Septiembre 2020

Aprobación: 10 Octubre 2020

\section{Resumen:}

El objetivo del presente estudio fue analizar el uso de las tecnologías de información y comunicación (TIC) en los procesos educativos y formativos de la educación secundaria. Para ello se diseñó y aplicó una investigación de campo de carácter descriptivo. El instrumento de recolección de información fue la encuesta. El universo estuvo constituido por cinco (5) instituciones de educación media, y la muestra por veinticinco estudiantes seleccionados de forma intencional. Las conclusiones más resaltantes: desconocimiento de las TIC por la mayoría del estudiantado; existe un número significativo de estudiantes que no participa en intercambios educativos utilizando las TIC; hay poca cultura en el uso de las TIC como herramienta de intercambio educativo, y se percibe disposición para estudiar las TIC como posibilidad educativa. Como recomendaciones: Sugerir el uso de las estrategias pedagógicas basadas en TIC en las diferentes áreas del saber y en las instituciones de formación en todos los niveles y modalidades, planificar y ejecutar cursos de capacitación sobre las TIC como herramientas para el mejoramiento de las actividades escolares. Se sugiere, como ideas para el debate, que las Tecnologías de Información y Comunicación incrementan el impacto que ejercen en diversos contextos, específicamente, en el educativo; profesorado y alumnado tienen a su disposición un amplio rango de posibilidades en cuanto a su uso.

Palabras clave: Formación educativa, Tecnologías de Información y Comunicación, TIC, Educación, Instituciones de educación media, Competencia digital.

\section{Abstract:}

In order to analyze how Information and Communication Technologies (ICT) impact secondary school educational processes, a study was designed and executed using a descriptive research methodology with data collected from the field through a survey. The universe for this study consisted of five (5) high schools with a sample of twenty-five purposefully selected students. Noteworthy findings include: Overall ignorance about ICTs among most of the surveyed students. Fewer students participating in educational interactions since they do not use ICTs at school. There is a general lack of awareness about the use of ICTs to enhance educational interactions. Yet, there was a perceived willingness to delve more into the benefits of using ICTs in education. Recommendations suggest developing ICT-based teaching strategies in all areas of learning and knowledge as well as develop teacher training courses that focus on ICTs as a mechanism for enhancing all school activities. Discussion points include accentuating the benefits of Information and Communication Technologies, specifically in educational contexts to provide a broad gamut of educational opportunities for both teachers and students.

KEYWORDS: Educational Processes, Information and Communication Technologies, ICT, Education, High Schools, Secondary Schools, Digital Skills. 


\section{INTRODUCCIÓN}

La formación y la educación secundaria son consideradas en la actualidad como una de las duplas más poderosas del saber, cuyo manto cubre el arte de la pedagogía en la producción de conocimientos, y el perfeccionamiento de las posturas didácticas. En este sentido, las instituciones educativas a nivel medio en Venezuela deben desarrollar nuevas competencias en el marco de las posibilidades digitales y tecnológicas que impactan la educación hoy día, destinadas a mejorar los procesos formativos en el desarrollo de las capacidades cognoscitivas y afectivas de los estudiantes, para aprender, desaprender y reaprender; es decir, aprender a aprender y para adaptarse a las nuevas exigencias de una sociedad en constante evolución.

Las lecturas que se realizan en los escenarios formativos, desde las perspectivas de esta nueva cultura, vale decir, en los procesos de aprendizajes en y desde las TIC, están siendo impactadas por las corrientes tecnológicas que hoy dominan las esferas académicas, y ese es un desafío que pernocta en las novedades didácticas y en las bondades que la pedagogía brinda a los que se están formando o aprendiendo. (Touron y Santiago, 2015).

Por otra parte, la educación tecnológica, más allá de ser una realidad, es una posibilidad cierta, que las instituciones educativas asumen de manera responsable, para atender los compromisos formativos que devienen de la práctica educativa. En esta coyuntura, las TIC vienen a sustituir los viejos esquemas expositivos que no responden a estatutos escolares modernos, y hoy la coyuntura es otra: ya las tecnologías son una realidad y una necesidad (Lorenzo-Lledó,Lorenzo-Lledóy Lledó Carreres, 2019).

De tal manera que, desde esta orientación, las temáticas que se ajustan a cada unidad curricular deben ser revisadas, planificadas y desarrolladas, de manera que sean digeribles para los estudiantes, más aún, sabiendo de las limitaciones de acceso a las tecnologías de información y de las posibilidades de realizar tareas de manera continua y de calidad.

... el sistema educativo se ve enfrentado a la necesidad de evolucionar para dar respuesta a las nuevas generaciones, permeadas por internet y con una alta utilización de tecnologías digitales en todos los ámbitos de su cotidianidad; generaciones que además, han desarrollado algunas destrezas particulares: accesan gran cantidad de información por fuera de la escuela, toman decisiones rápidamente, tienen una gran capacidad de procesamiento paralelo, son altamente multimediales y aprenden de manera diferente (Zabala Vargas,Zabala Vargas,Lizcano Dallosy Lizcano Reyes,2017,p. 68).

De allí que el objetivo principal de este trabajo es analizar el uso de las tecnologías de información y comunicación (TIC) en los procesos educativos y formativos de la educación secundaria, de acuerdo con los desafíos que hoy se deben salvar en esta materia. Como propósitos específicos: identificar las herramientas o tecnologías de comunicación utilizadas por los estudiantes en sus actividades educativas a nivel de educación media; describir las condiciones de equipamiento tecnológico por parte de los estudiantes en los procesos formativos en los Liceos; precisar el nivel de manejo de las herramientas tecnológicas en los intercambios académicos por parte de los estudiantes; y describir los intercambios educativos mediante el uso de las TIC por parte de los estudiantes.

Para ello se seleccionaron cinco (5) instituciones de educación media, donde se realizó la aplicación del instrumento de recolección de datos. Se trata de un proceso de ayuda continua y sistemática dirigido a los estudiantes en todos sus aspectos, con la finalidad de potenciar el desarrollo humano y en los aspectos tecnológicos a lo largo de su escolaridad, debido a esto, la acción orientadora (y pedagógica) debe extenderse a todas las asignaturas (Pochulu,Font y Rodríguez, 2016).

La educación desde sus diferentes concepciones desarrolla protocolos formativos que son impulsados por las nuevas tecnologías (European Agency for Safety and Health at Work, 2018). Esta orientación sobre los nuevos escenarios de aprendizaje de las TIC, debe considerar de manera integral lo cognitivo y afectivo, más precisamente, a partir de lo emocional se construye un escenario motivador, para que las nuevas tecnologías tengan espacio en las instituciones educativas a nivel medio. "Hasta finales del siglo pasado, la educación 
centraba su interés principalmente en los aspectos cognitivos, quedando reducidos al ámbito privado, lo social y emocional...”. (Porras, Pére, Checa y Luque, 2020, p. 3).

El presente trabajo está estructurado de la siguiente manera: el resumen, donde se expone de manera sucinta lo que se trata en la investigación; la introducción, que describe los temas, objetivos y presentación del trabajo; estado de la cuestión, aquí se hace referencia de investigaciones y teorías educativas y las TIC; la metodología, que orienta este estudio; resultados y discusión, presentados en forma de tablas; población y muestra, donde se explica el universo y la forma de escogencia de los sujetos a quienes se le aplicó el instrumento de investigación; conclusiones, resultados puntuales del estudio; recomendaciones e ideas para el debate.

\section{EsTADo DE LA CUESTIÓN}

La integración de las actividades educativas a través de las TIC son una posibilidad extraordinaria en el presente milenio. No solo se integra tecnología, sino que se adicionan ideas, proyectos y posibilidades (Angelini, 2016). Por tanto, estudiar los impactos de las tecnologías en la educación es un escenario que depara muchas aristas para debatir y orientar los procesos educativos hacia otros escenarios.

Arévalo,Gamboa, y Hernández (2016) destacan en su trabajo la importancia de la educación a nivel de secundaria en cuanto al uso de las TIC, pues se trata de una herramienta que facilita la adquisición de los aprendizajes, así como el intercambio efectivo y en tiempo real con compañeros y profesores.

No disponer de herramientas tecnológicas en la actualidad es condenarse al atraso, es realizar un ejercicio regresivo indetenible, pues la tecnología tiene una evolución excesivamente vertiginosa, y lo que hoy es una realidad, en pocos días, ya es cuestión del pasado. De allí que los Liceos, deben obrar de forma proactiva y superar las deficiencias en cuanto a las inversiones en esta área, pero que sea un proyecto educativo insertado en la cultura digital que hoy impacta las instituciones educativas.

Las TIC han tenido muchas formas de enfocarse y justificarse, una de ellas es la educación a distancia, que, justamente, el uso de herramientas tecnológicas facilita las actividades, más aún en las circunstancias actuales, donde hay restricciones de movilidad por la pandemia del Covid-19. El trabajo realizado por Vasilevska, Rivza, Pivac, Alekneviciene, y Parlinska (2017) destaca la necesidad de implementación de políticas educativas, que den espacio a todos los estudiantes que opten por seguir sus estudios universitarios, y precisamente esta nueva filosofía de educación a distancia utilizando tecnologías de punta es una apuesta que los autores sugieren en su trabajo.

Las tecnologías de información y comunicación también son abordadas por Márquez (2016) en su trabajo, cuyo análisis parte de las tremendas diferencias que se dan en los diferentes estratos sociales, y las posibilidades de brindar alternativas educativas a sectores excluidos y que se deben implementar en las reformas educativas que se dictaminen para tal fin. Se trata de dar espacios a quienes tienen condiciones desventajosas, y cuentan con recursos escasos para estudiar.

En la tónica de la educación en línea o de la cultura escolar digital, el trabajo realizado por Kumar, Kumar, Palvia y Verma, (2017) refiere precisamente que se trata de una opción plausible, posible y realizable, en la actualidad la pertinencia del uso de la cultura digital en la educación tiene una pertinencia coyuntural, y en esto el uso de la TIC sugiere una nueva concepción en lo educativo y formativo a nivel mundial.

Una investigación realizada por López-Vélez (2018) destaca que la educación en todos los niveles y modalidades en la actualidad debe convertirse en el escenario ideal para potenciar los procesos de formación de los estudiantes, este hecho se debe consustanciar con los avances científicos y tecnológicos que hoy invaden los espacios académicos. Para ello, el uso de las tecnologías es vital, y este elemento se constituye en un aspecto determinante y a veces definitivo en las instituciones educativas.

La educación como proceso transformador debe estar en consonancia con los principios fundamentales de la formación de los estudiantes; Luévano, López y Orrante, (2020), en su trabajo, entre otros aspectos, 
afirman que: "La educación genera las condiciones de desarrollo social y económico que impulsan las economías locales y contribuyen a reducir las brechas de pobreza y desigualdad; es el catalizador más efectivo para desarrollar, potencializar y transmitir las habilidades, conocimientos...”. (p. 3).

La educación, para su práctica hace uso de diferentes recursos, entre ellos los tecnológicos, que vienen a complementar la multiplicidad de opciones en cada estrato educativo (Balcaza, Contreras y Font, 2017). El docente en estas latitudes deja de ser un buscador de nuevas competencias para enseñar y adquirir conocimientos, abierto a nuevas técnicas direccionales de enseñanza, da un carácter singular en los procesos formativos. Esa búsqueda incesante de conocimientos orientados a mejorar la práctica pedagógica, anima a docentes y estudiantes, a permear teorías que describen la acción escolar, como si se tratara de una norma y no de un intercambio de saberes.

Otro trabajo interesante es el presentado por Balladares-Burgos (2018), cuyo debate se centra en la inclusión de las personas en el acceso a las tecnologías de información y comunicación. Critica que no todas las personas cuentan con los recursos para tener posibilidades reales a las tecnologías que circulan en las redes, de allí su preocupación por una educación digital inclusiva.

Pese a ello, y sobre todo a los cambios paradigmáticos que se han venido suscitando en el contexto cultural contemporáneo, la educación venezolana sigue caracterizándose por el predominio de elementos tales como: la fragmentación de saberes, el estudio empírico del entorno social, conocimiento parcial y analítico de los hechos, poca importancia a la historicidad de los acontecimientos sociales, exclusión y limitaciones en el acceso a la tecnología, entre otros.

En este orden, los autores Escobar, Restrepo, y Castrillón (2016), en su investigación abordan esta temática, similares al autor antes citado, discuten la pertinencia de las tecnologías actuales como una posibilidad real de inclusión en todos los aspectos: laborales, industriales, educativos, entre otros; se apuesta a la no discriminación, sino a una educación actualizada e inclusiva. Asimismo, Watts y Lee (2017) exponen en su trabajo la posibilidad real de construir un mundo donde las posibilidades, a partir del manejo de las TIC, se transforme en un espacio para mejorar la calidad de vida y la creación de conocimientos de manera inclusiva.

Reyes y Prado (2020), en su trabajo, concluyen, entre otros aspectos interesantes, lo siguiente: "En síntesis, las TIC son herramientas determinantes e indispensables para acercar democráticamente la educación a todas aquellas personas que se encuentran en situación de vulnerabilidad permitiendo su inclusión en la sociedad del siglo XXI [...]" (p. 16).

El aprendizaje, entendido como los cambios de conducta que experimentan los sujetos, se debe en buena medida por el trato de los contenidos que realizan los docentes, de allí que su formación debe estar consustanciada con los principios y valores educativos en los niveles intermedios, donde los estudiantes están en su etapa de adolescencia, pero deben ser motivados para el cumplimiento de sus responsabilidades, y en ello se incluye el uso de las herramientas digitales (Mallart, Font Y Malaspina, 2015).

Por tanto, cuando se habla de la formación en el contexto de las TIC o de las aulas virtuales, se refieren a la aplicación de una pedagogía científica, epistemológica, que establezca, desde los contextos actuales, las posibilidades educativas de los estudiantes, entendiendo que son la razón y esencia de la educación media y su futuro.

\section{MÉTodo}

La presente investigación está circunscrita dentro de los estudios de campo, toda vez que se realizó en el propio lugar donde los estudiantes cursaron sus estudios. Según el Manual de Trabajos de Grado de Especialización y Maestrías y Tesis Doctorales (2006), de la Universidad Pedagógica Experimental Libertador:

Se entiende por Investigación de Campo, el análisis sistemático de problemas en la realidad, con el propósito bien sea de describirlos, interpretarlos, entender su naturaleza y factores constituyentes, explicar 
sus causas y efectos, o predecir su ocurrencia, haciendo uso de métodos característicos de cualquiera de los paradigmas o enfoques de investigación conocidos o en desarrollo [...] (p. 11).

El nivel de la investigación es descriptivo, pues de los resultados obtenidos se pudo caracterizar la población, así como los elementos constitutivos de acuerdo con los objetivos y propósitos del estudio.

La población estuvo conformada por los estudiantes que cursaron estudios a nivel de quinto año de bachillerato, último grado para optar a los estudios a nivel universitarios, en los siguientes Liceos: Francisco Isnardi, Miguel José Sanz, Félix Ángel Lozada, Félix Armando Núñez y Nuevos horizontes, de la ciudad de Maturín, Venezuela. La muestra estuvo constituida por veinticinco (25) estudiantes, cinco (5) de cada Liceo, que, para los efectos del estudio, se escogieron de acuerdo con el muestreo no probabilístico intencional, que según Estrada (2019a):

Se realiza cuando la selección de la muestra se ejecuta 'a conveniencia' del investigador, esto porque le interesa un determinado grupo, con ciertas y determinadas características o cualquier otro requisito, que, para los efectos del estudio, el investigador estime sean los más adecuados” (p.13).

\section{Instrumento de recolección de información}

Para la recolección de datos se aplicó la encuesta, porque se trata de un instrumento versátil y de fácil aplicación; asimismo, se expresan en tablas que contienen los datos absolutos y porcentuales, que permiten una mejor apreciación de lo que se estudia. "Una encuesta es una técnica cuantitativa que consiste en una investigación realizada sobre una muestra de sujetos, representativa de un colectivo más amplio, que se lleva a cabo en el contexto de la vida cotidiana...”. (Estrada, 2019a, p. 27).

Ventajas de la encuesta:

- Técnica más utilizada y que permite obtener información de casi cualquier tipo de población.

- Permite obtener información sobre hechos pasados de los encuestados.

- Gran capacidad para estandarizar datos, lo que permite su tratamiento informático y el análisis estadístico.

- Relativamente barata para la información que se obtiene con ello. (Estrada, 2019a)

De tal manera que se trata de un instrumento de fácil aplicación y de obtención de resultados de manera práctica. En este orden, es importante precisar que los instrumentos de investigación, así como las técnicas que se aplican, deben tener la consistencia adecuada para garantizar resultados confiables o ajustados a la perspectiva fenomenológica del problema que se indaga, que en este caso es Formación educativa en y desde las TIC en educación media: el reto de hoy. Esas características son la validez y la confiabilidad. En el primer caso, se determinó aplicando el criterio de Juicio de Expertos, en este sentido, este fue revisado y evaluado por los doctores Fillipo Ricciardi, Ezequiel Zurita y Yusmilys Gallardo, y luego de la revisión respectiva sugirieron las observaciones pertinentes, afirmando que la encuesta presentada se ajusta a los parámetros para su aplicación, y en específico en este estudio.

En cuanto a la confiabilidad, se aplicó el Coeficiente de coincidencias T.= $1-$ R./TR, donde R.son las respuestas coincidentes, y TR son las respuestas totales (Estrada, 2019b). En este caso se escogió una muestra piloto de 10 estudiantes del mismo universo, pero no coincidentes con la muestra seleccionada, y se determinó que $\mathrm{T} .=0,89$; se trata entonces de un instrumento con una consistencia muy buena para los efectos de la presente investigación.

Luego de la aplicación del instrumento se contabilizaron los resultados y se expresaron en tablas de doble entrada de manera absoluta y porcentual, para facilitar el análisis descriptivo e inferencial que se derivan de los hallazgos. 


\section{RESULTADOS Y DISCUSióN DE HALLAZgos.}

A continuación, se presentan los resultados obtenidos, producto de la aplicación de la encuesta a la muestra seleccionada:

TABLA 1

Participación de los estudiantes en cursos o talleres sobre las TIC.

\begin{tabular}{l|l|l|l}
\hline Ittems & Opción & Frecuencia & $\%$ \\
\hline $\begin{array}{l}\text { ¿Participas en actividades ocursos } \\
\text { de formación sobre las TIC? }\end{array}$ & Mucho Poco Muy poco Nunca & 26107 & 824 \\
& & & \\
& Total & 25 & 100 \\
\hline
\end{tabular}

Fuente: Encuesta aplicada a los estudiantes, mayo de 2020.

De los resultados expresados en la Tabla 1, se aprecia que el 68\% de los estudiantes participan muy poco o nunca lo hace en cursos o actividades sobre tecnologías de comunicación e información, es decir, no poseen ni desarrollan las capacidades para atender las diferentes posibilidades de formación utilizando las TIC.

En relación con este ítem, el 24\% de los encuestados afirman no haber realizado nunca cursos de formación sobre las TIC.

Solo el 8\% de ellos manifiesta conocer con cierta propiedad estas tecnologías. El problema se acrecienta con el complemento, que es la mayoría, y manifiestan no estar en sintonía con estos avances.

Por ello, es necesario trabajar con las TIC de manera didáctica y no solo instrumental, esto es, generar una metodología que permita proponer actividades en las que estos recursos estén presentes, aprovechando sus potencialidades las que trascienden la simple transmisión de información, sino que aportan o contribuyen a la generación de conocimiento [...](González, 2018,p. 30).

TABLA 2

Con qué frecuencia utilizas el computador e internet para cumplir con tus tareas y trabajos.

\begin{tabular}{l|l|l|l}
\hline Items & Opción & Frecuencia $\%$ \\
\hline $\begin{array}{l}\text { Con qué frecuencia utilizas el } \\
\text { computador e internet para cumplir con } \\
\text { tus tareas y trabajos. }\end{array}$ & Siempre Casi siempre A veces Nunca & 36124 & 12244816 \\
\hline
\end{tabular}

Fuente: Encuesta aplicada a los estudiantes, mayo de 2020.

Como el propósito de esta investigación es analizar el uso de las TIC en los procesos educativos y formativos, vale la pena resaltar, de acuerdo con los resultados que se muestran en la Tabla 2, que 64\% muy pocas veces o nunca utiliza el internet para cumplir con sus tareas rutinarias, es decir existe una debilidad muy marcada que debe ser atendida a corto plazo. En contraste, el 12\% manifiesta hacerlo con mayor frecuencia.

Con cierta frecuencia, el 24\% de los estudiantes afirman que utilizan el computador e internet para realizar y enviar sus tareas. Las condiciones de aislamiento que se da en las mayorías de los países y grandes ciudades, se está ante un resultado preocupante, pues la mayoría de las obligaciones académicas se realizan en línea. Y si esta es la realidad, el panorama es crítico.

Tabla 3

Realizas tareas de navegación 
TABLA 3

Realizas tareas de navegación

\begin{tabular}{|c|c|c|c|}
\hline Items & Opción & Frecuencia & $\%$ \\
\hline $\begin{array}{l}\text { Realizas tareas básicas denavegación por la } \\
\text { Redcomo: guardar direcciones en marcadores o }\end{array}$ & Mucho Poco Muy poco Nunca & -8152 & 032608 \\
\hline navegación, enviar y recibir correos, entre otras & Total & 25 & 100 \\
\hline
\end{tabular}

Fuente: Encuesta aplicada a los estudiantes, mayo de 2020.

Es una reafirmación con respecto a los resultados de las tablas anteriores. En la Tabla 3, los estudiantes manifiestan, en un 68\%, que muy poco o nunca navegan o intercambian correos electrónicos; más precisamente, esta desviación, que significa el no manejo de las tecnologías, da lugar a especulaciones sobre los problemas educativos y de formación en las instituciones involucradas en el estudio.

“[...] La prevalencia de las TIC en la sociedad del presente y del futuro demandará sin lugar a dudas de una respuesta adecuada en el campo investigador, [...]” (Lorenzo-Lledó, Lorenzo-Lledó y Lledó Carreres, 2019, p. 304), por eso las investigaciones en este ámbito deben ser consecuencia de una realidad que impacta a la sociedad y a la educación.

Tímidamente, en los resultados se percibe que el 32\% manifiesta el manejo de las redes sociales, como, por ejemplo, enviar y recibir correos, guardar direcciones, entre otros. Es posible que estas instituciones no hayan actualizado sus plataformas, o ha sido muy poca la inversión que se ha realizado al respecto.

TABLA 4

Valoración del servicio de internet residencial de los estudiantes.

\begin{tabular}{|c|c|c|c|}
\hline Items & Opción & Frecuenci & $\%$ \\
\hline \multirow[t]{3}{*}{ El servicio de internet en tu hogar, lo evalúas como } & Excelente Bueno Con interrupciones & -416 & 01664 \\
\hline & No tengo & 5 & 20 \\
\hline & |Total & 25 & |100 \\
\hline
\end{tabular}

Fuente: Encuesta aplicada a los estudiantes, mayo de 2020.

Según la Tabla 4, el servicio de internet es casi una necesidad y una preocupación de los padres por mantenerse informados. En el caso de los estudiantes consultados, el 64\% afirma tener el servicio pero con interrupciones, es decir, es de mala calidad, y para la realización de tareas se transforma en un ruido importante, que interrumpe el desarrollo adecuado de los estudios al utilizar las TIC.

No obstante, existe un $20 \%$ que no posee este servicio, es decir, que prácticamente está en desventaja con sus compañeros de estudios. Este tipo de limitaciones pudieran subsanarse, si se contara con suficientes centros de comunicación y de acceso a internet, que no es el caso. Solo el 16\% afirma tener buen servicio, y para los efectos, es un grupo menor comparado con el total.

Muchas de las limitaciones que se presentan en los intercambios de aprendizajes son las condiciones en que los sujetos de aprendizaje se encuentran; si las posibilidades son parecidas, los niveles de aprendizaje son significativos, en caso contrario se da una especie de discriminación (Kwan, MacLeod, Chandler y Fox, 2017). Y esto es lo que sucede con este último grupo de estudiantes: si no existen las condiciones mínimas para imbuirse en las TIC, tendrán serias limitaciones en sus estudios. 
TABLA 5

Importancia de la implementación de herramientas en la web

\begin{tabular}{l|l|l|l}
\hline Items & Opción & Frecuencia \\
\hline $\begin{array}{l}\text { Consideras importante la } \\
\text { implementación de las Herramientas } \\
\text { de la Web como estrategias de } \\
\text { enseñanza }\end{array}$ & Mucho Poco Muy poco No opino & $1942-$ & 761680 \\
\hline
\end{tabular}

Fuente: Encuesta aplicada a los estudiantes, mayo de 2020.

De acuerdo a la Tabla 5, la gran mayoría de los estudiantes, el 76\%, considerala implementación de herramientas de la web, y es una opinión que tiene relevancia, sea por la necesidad coyuntural que se vive, o bien por la apertura que se asume y ensancha en el mundo tecnológico y en los sistemas de comunicación y la tecnología.

La educación virtual se ha convertido en una poderosa alternativa para los estudiantes autodidactas que quieren alcanzar sus logros académicos y potenciar su proceso de aprendizaje basándose en la autonomía y la responsabilidad. Partiendo de la premisa de que los educadores están a cargo de mediar y facilitar el proceso de enseñanza-aprendizaje, es absolutamente necesario que los estudiantes de la modalidad virtual cuenten con materiales dinámicos y estructurados. (Muñoz Oyola, y Celis Vargas, 2017, p. 39).

Lo cual se debe considerar de manera reiterativa; en este sentido, el complemento, el 24\%, no se siente identificado con esta herramienta como estrategia de enseñanza, pues opinan que le dan poca o muy poca importancia. Sin embargo es una realidad que las TIC siguen impactando a las instituciones y a la educación de manera particular, tan es así que su uso es muestra de avance y progreso tecnológico (García-Peñalvo, 2019).

TABLA 6

Utilizas recursos tecnológicos en el proceso de formación

\begin{tabular}{l|l|l|l}
\hline Items & Opción & Frecuencia $\%$ \\
\hline $\begin{array}{l}\text { Utilizas recursos tecnológicos en el } \\
\text { proceso de formación y comunicación }\end{array}$ & Mucho Poco Muy poco Nunca & -2221 & 088084 \\
& Total & 25 & 100 \\
\hline
\end{tabular}

Fuente: Encuesta aplicada a los estudiantes, mayo de 2020.

Este resultado contrasta con lo expresado en la Tabla 6, y es la realidad que describen los estudiantes de las instituciones involucradas. Como se percibe, el $96 \%$ afirma que utiliza poca o muy pocas veces los recursos tecnológicos y de información, y esto es preocupante, pues hay una realidad que impacta todos los escenarios: la tecnología y las TIC siguen un avance impresionante, lo que significa que se corre el peligro de que esta masa de estudiantes quede peligrosamente rezagada. En este orden, el $4 \%$ opina que nunca los utiliza.

El campo de la tecnología aplicada a la educación tiene su desarrollo a la par de la misma tecnología, esto ha dado lugar a nuevas formas de aprendizaje y de enseñanza. Para los inmigrantes digitales representa un reto mayor, gran parte de las generaciones con poca estimulación de las nuevas tecnologías, presentan dificultades para dominarlas, mermando el desarrollo de las habilidades necesarias para la comunicación y generación de contenidos digitales. Las generaciones de jóvenes alumnos nacidos en las últimas dos décadas, presentan mayor interés por el uso de la tecnología. Han cambiado sus esquemas de pensamiento y organización, así como su forma de comunicación (Rubín-Ruiz, Chávez-Melo, 2017, p. 15).

Y esta es una de las bondades de estudios como el que se realiza, pues permite caracterizar y describir a los sujetos de investigación, de acuerdo con sus opiniones y posiciones sobre temas como el que se indaga. Esto, por añadidura, permite hacer los correctivos curriculares y académicos pertinentes. 
TABLA 7

Participación en cursos de capacitación en recursos tecnológicos en el proceso de formación

\begin{tabular}{l|l|l|l|l}
\hline Items & Opción & Frecuencia \\
\hline $\begin{array}{l}\text { Participarís en cursos de } \\
\text { capacitación en el manejo de } \\
\text { recursos tecnológicos para } \\
\text { fortalecer tu formación }\end{array}$ & Totalmente de acuerdo De acuerdo En desacuerdo En total desacuerdo & $232 \ldots$ & 92800 \\
\hline
\end{tabular}

Fuente: Encuesta aplicada a los estudiantes, mayo de 2020.

Este resultado se orienta a la necesidad que expresan los integrantes de la muestra, en el sentido de tener la disposición de participar en cursos de capacitación para fortalecer sus conocimientos sobre las TIC. "En una sociedad asentada cada vez más en el conocimiento, y cuando las Tecnologías de la Información y las Comunicación (TIC) se tornan más asequibles a todos en tiempo real, [...]" (Acosta Estrada y Díaz Gispert, 2015, p. 109). En efecto, según la Tabla 7, el 100\% expresa que está de acuerdo o totalmente de acuerdo en ser partícipe de este tipo de actividades académicas, lo cual es menester reafirmarlo de manera prolija.

Esto implica, entre otras interpretaciones, que hay una tendencia positiva por el estudio y conocimiento de las tecnologías como herramientas de intercambios educativos, lo cual es plausible.

Estas reflexiones reafirman la importancia de las tecnologías de información y comunicación en la educación contemporánea. Se trata, en definitiva, de una herramienta que permite hacer del acto escolar, una experiencia significativa (Fernández-Cruz, y Fernández-Díaz, 2018).

\section{Conclusiones}

En aras de dar respuestas a los objetivos de la investigación, específicamente en lo atinente a precisar el nivel de manejo de las herramientas tecnológicas en los intercambios académicos, se observa un marcado desconocimiento sobre las TIC por parte de los estudiantes de las instituciones involucradas en el estudio, esto efectivamente limita las posibilidades formativas, dadas las condiciones especiales que se vive en la actualidad. La evolución de las tecnologías de información y comunicación, específicamente a finales de la segunda década del presente siglo, han impactado los diferentes espacios de la actividad educativa. Las instituciones escolares han tenido que experimentar rigurosas transformaciones en lo didáctico y pedagógico, para ajustarse a escenarios, no solo novedosos, sino necesarios, que impactan los procesos de formación a nivel global.

Existe un conocimiento muy generalizado y vago de las TIC y su utilidad en los procesos educativos hoy en día, no así su manejo adecuado. Lo que se transforma en una desventaja considerable. Esto da significado al objetivo que trata la descripción de las condiciones de equipamiento tecnológico en los procesos formativos de los estudiantes de educación media, de allí la relevancia de las tecnologías en el ámbito educativo en el presente milenio.

Con respecto a los intercambios educativos mediante el uso de las TIC por parte de los estudiantes, un número significativo de ellos no participan en estos; se trata de una realidad que afecta su prosecución escolar y los éxitos futuros en materia académica.

Hay poca cultura en el uso de las TIC como herramienta de intercambio educativo, de tal manera que se debe explorar con más detenimiento esta debilidad, a fin de establecer criterios que permitan hacer un diagnóstico profundo, y así proponer estrategias alternativas que subsanen la situación descrita. Esto implica que dentro de las instituciones educativas de educación media, donde se realizó esta investigación, urge una transformación, en primer lugar, en lo tecnológico y, en segundo término, en lo cultural, pues la digitalización 
o el uso de las TIC ya es una realidad impostergable, y no una apreciación vacua o intrascendente. Es por ello, que los Liceos, desde sus perspectivas pedagógicas y académicas, deben impulsar tales cambios.

Se percibe una disposición a estudiar e informarse sobre las TIC como elementos coadyuvantes en los procesos educativos y formativos. Lo que abre un espacio interesante para propiciar escenarios académicos y de intercambio de ideas, que posibiliten su conocimiento y manejo de herramientas tecnológicas, hoy en día vitales para acceder a espacios superiores. Aunque eventualmente se ha podido inferir que el uso de las TIC en los intercambios educativos docentes-estudiantes es una posibilidad cierta, existen algunas limitaciones por las carencias de habilidades y manejo adecuado de herramientas digitales, orientadas a acortar distancias y mejorar los procesos educativos en las instituciones de educación media.

\section{Recomendaciones}

Como una forma de dar una opción plausible a los estudiantes en materia de las TIC, así como para cerrar esta investigación, en cuanto a tareas futuras, se exponen las siguientes recomendaciones:

- Sugerir el uso de las estrategias pedagógicas basadas en TIC en las diferentes áreas del saber y en las instituciones de formación en todos los niveles y modalidades. Esto puede favorecer el interés, y aumentar la motivación a su uso.

- Planificar y ejecutar cursos de capacitación sobre las TIC como herramientas para el mejoramiento de las actividades escolares en estudiantes de educación media. "[...] Se refiere, por tanto, a la oportunidad de un aprendizaje transformacional que cambia perspectivas”. (Tamarony, 2020, p. 53).

- Priorizar, desde la concepción didáctica el uso de las TIC para mejorar los procesos comunicacionales y de aprendizaje en las instituciones educativas a nivel medio. Es decir, la implementación de este tipo de herramientas, dan versatilidad y manejo rápido a las informaciones.

- Un desafío para las próximas décadas, es consolidar un sistema escolar que forme y eduque para la vida, para la libertad, para trascender de los espacios tradicionales y monótonos a estadios de trascendencia y posibilidades que traten de aproximarse a un sistema educativo ajustado a las nuevas realidades. Por eso, las TIC son, en su conjunto, un bloque de posibilidades significativas, que invita a explorar ese universo disperso de galaxias cognitivas, que impulsan su indagación y estudio. De eso se trata, de seguir, de no detenerse, y de conquistar espacios educativos, más allá de los estándares curriculares, sino que sea una ventana para irrumpir en otros estadios y otras posibilidades. "La eficiencia de los profesionales constituye la clave del éxito, que permite la optimización de los procesos en todos los departamentos que conforman una organización, [...]" (Fuentes, 2020, p. 53).

\section{Ideas para el debate}

En el devenir que se vive, es menester puntualizar algunos escenarios posibles, entre ellos, los que incluyen los sistemas de aprendizaje en línea (Sarsa, 2015), se sugiere la implantación y capacitación en los sistemas educativos digitales, donde los trabajadores, estudiantes y maestros, así como los liceos, asuman con conciencia crítica esta nueva perspectiva cultural.

Con el uso de las TIC se pueden elaborar distintos materiales (vídeos, laboratorios virtuales, artículos docentes, etc.), entre los que cabe destacar un tipo con una estructura muy concreta: los objetos de aprendizaje. Se trata de materiales didácticos en cualquiera de estos formatos, cuyas características diferenciadoras se citan a continuación:

- Son de carácter pedagógico.

- Se trata de materiales originales.

- Su contenido es interactivo (las explicaciones complementan a la imagen, se intercalan interrogaciones y refuerzos motivadores, se incluye ejemplos y contraejemplos).

- Tiene sentido en sí mismo, puesto que no depende de otros objetos para su comprensión y aprendizaje.

- Tiene una extensión de entre 5 y 10 minutos aproximadamente.

. Es indivisible. 
- Puede reutilizarse en distintos contextos educativos (no está contextualizado en una asignatura o unidad didáctica, tiene licencia creative commons). (Roldán-Blay, Escrivá-Escrivá, Fuster-Roig, Segura-Heras, y Roldán-Porta, 2018, p. 395).

Entre otras posibilidades y retos, se abre la discusión acerca de las TIC y su impacto en los procesos educativos y formativos, de donde se decanten los futuros profesionales que den cuenta de una sociedad moderna y condiciones de vida favorables en todos los ámbitos.

\section{REFERENCIAS BIBLIOgRÁFICAS}

Acosta Estrada, L. A. y DíazGispert, I (2015). Desafíos de la Universidad de Otavalo en el siglo XXI. Revista Estrategia y Gestión Universitaria,3(2), 108-119, http://revistas.unica.cu

Angelini, M. (2016). Integration of the Pedagogical Models 'Simulation' and 'Flipped Classroom', en Teacher Instruction, 1, 1-8. doi: https://doi.org/10.1177/2158244016636430

Arévalo, M. A., Gamboa, A. A. y Hernández, C. A. (2016). Políticas y programas del sistema educativo colombiano como marco para la articulación de las TIC. Aletheia, $8(1), 12-31$. Recuperado de https://aletheia.cinde.org.co /index.php/ALETHEIA/article/view/302

Balcaza, T.; Contreras, A. y Font, V. (2017). Análisis de libros de texto sobre la optimización en el bachillerato, Bolema, 31(59), 1061-1081. doi: http://dx.doi.org/10.1590/1980-4415v31n59a11

Balladares-Burgos, J. (2018). Competencias para una inclusión digital educativa. Revista PUCE, (107), 191-211. Recuperado de http://www.revistapuce.edu.ec/index.php/revpuce/article/view/179/261

Estrada, J. (2019a). Notas de estadistica. Maturín: Ediciones Fumaprif.

Estrada, J. (2019b). Evaluación de los aprendizajes. Maturín: Ediciones Fumaprif.

Escobar, C., Restrepo, A. y Castrillón, A. (2016). Más allá de la brecha digital: la apropiación social de las tecnologías de la información y la comunicación (TIC) como opción para la inclusión. En B. Muñoz y A. Barrantes. (Ed). Equidad e inclusión social: superando desigualdades hacia sociedades más inclusivas (pp. 185-201). Washington: Organización de los Estados Americanos OEA. Recuperado de https://bit.ly/3bClkpb

European Agency for Safety and Health at World. (2018). WhatisOiRA? Recuperado de https://oiraproject.eu/es/ what-oira

Fernández-Cruz, F.J., y Fernández-Díaz, M.J. (2018). El proceso de integración y uso pedagógico de las TIC en los centros educativos madrileños. Educación XX1, 21(2), 395-416, doi: 10.5944/educXX1.17907

Fuentes, J. (2020). La supervisión transformadora en el Instituto Nacional de Capacitación y Educación Socialista (INCES) en el Estado Delta Amacuro. Revista Multidisciplinaria Saber Universitario, (3), 69-78. Recuperado de https://saber755.webnode.com.ve/_files/200000012-d44eed44f0/Saber\%20Universitario\%203\%20-\%202 020-4.pdf

García-Peñalvo, F.J. (2019). Education in the Knowledge Society PhD Programme 2019 Kick-off Meeting. Salamanca, España. Recuperado de https://bit.ly/33kfJzl

González, C. (2018). Contribuciones de los usos didácticos de las TIC para la formación literaria de jóvenes lectores. Aula de Encuentro, 20(2), 21-34. Recuperado de https://revistaselectronicas.ujaen.es/index.php/ADE/article/ view/3946/pdf

Kumar, A., Kumar, P., Palvia, S. y Verma, S. (2017). Online education worldwide: Current status and emerging trends. [Educación en línea en todo el mundo: Estado actual y tendencias emergentes].Journal of Information Technology Case and Application Research, 19(1), 3-9. doi:10.1080/15228053.2017.1294867

Kwan, E., MacLeod, S., Chandler, M. y Fox, T. (2017). Report on a literature review of reforms related to the 2006 European Framework of Key Competences for lifelong learning and the role of the Framework in these reforms. European Commission, Bruselas. Recuperado de https://bit.ly/2Ixsj5F

Luévano, K., López, A., Orrante, M. (2020). Diagnóstico para nueva oferta educativa a distancia: estudio de caso basado en expectativas educativas. Revista Educación,44(2). doi: 10.15517/revedu.v44i2.39330 
López-Vélez, A. L. (2018). La escuela inclusiva. El derecho a la equidad y a la excelencia educativa. Universidad del País Vasco, Bilbao, https://addi.ehu.es/bitstream/handle/10810/26837/USPDF188427.pdf?sequence=1

Lorenzo-Lledó, G.; Lorenzo-Lledó, A.; Lledó Carreres, A. (2019). Las TIC en el contexto educativo a través de la producción científica en español. Revista General de Información y Documentación,29(2), 287-307.Recuperado de https://rua.ua.es/dspace/bitstream/10045/100529/1/2019_Lorenzo-Lledo_etal_RevGenInfDoc.pdf

Mallart, A., Font, V. y Malaspina, U. (2015). Reflexión sobre el significado de qué es un buen problema en la formación inicial de maestros. Perfles educativos, 38(152), 14-30.doi:https://doi.org/10.22201/iisue.24486167e.2016.15 2.57585

Márquez, A. (2016). La desigualdad social y las reformas educativas. En A. Márquez. (Ed.), Perfiles Educativos. México: Instituto de Investigaciones sobre la Universidad y la Educación IISUE-UNAM. Recuperado de https://www .iisue.unam.mx/perfiles/articulo/2016-154.pdf

Muñoz-Oyola, J. E. y Celis-Vargas, D. M. (2017). Diseño y evaluación de objetos virtuales de aprendizaje (OVA) fundamentados en el método "Enseñanza de la lengua basada en tareas (Task-Based Language Teaching-TBLT)". Memorias CIMTEDXXII Congreso Internacional sobre Educación Bimodal .Competencias Digitales, Innovación y Prospectiva”, p.p. 36-52. Medellín, Colombia. Recuperado de https://bit.ly/37LNiiX

Pochulu, M., Font, V. y Rodríguez, M. (2016). Desarrollo de la competencia en análisis didáctico de formadores de futuros profesores de matemática a través del diseño de tareas. Revista Latinoamericana de Investigación en Matemática Educativa-RELIME, 19(1), 71-98. doi: http://dx.doi.org/10.12802/relime.13.1913

Porras, S., Pérez, C., Checa, P. y Luque, B. (2020). Competencias emocionales de las futuras personas docentes: un estudio sobre los niveles de inteligencia emocional y empatía. Revista Educación,44(2). doi: 10.15517/ revedu.v44i2.38438

Roldán-Blay, C., Escrivá-Escrivá, G., Fuster-Roig, V., Segura-Heras, I. y Roldán-Porta, C. (2018). Utilización de videos screencast para la mejora del aprendizaje de teoria de circuitos en grados de ingeniería. XXVI Congreso Universitario de Innovación Educativa En las Enseñanzas Técnicas, Escuela Politécnica de Ingeniería de Gijón. Universidad de Oviedo. Recuperado de https://bit.ly/3smeNHh

Reyes, R., y Prado, A. (2020). Las Tecnologías de Información y Comunicación como herramienta para una educación primaria inclusiva. Revista Educación, 44(2). doi: 10.15517/REVEDU.V44I2.38781

Rubín Ruiz, S. y Chávez Melo, G. (2017). Experiencias académicas para el desarrollo de la literacidad digital en el Posgrado. Memorias CIMTEDXXII Congreso Internacional sobre Educación Bimodal Competencias Digitales, Innovación y Prospectiva., p.p. 13-22. Medellín, Colombia. Recuperado de https://bit.ly/2G4MU3H

Sarsa, J. (2015). Influencia en el aprendizaje de la aplicación de un sistema online de distribución de contenidos multimedia y streaming de video (Tesis doctoral). Universidad de Zaragoza, Zaragoza. Recuperado de https://dialnet.uniri oja.es/servlet/tesis?codigo $=133114$

Tamarony, Y. (2020). La gestión comunicacional en el proceso gerencial de las instituciones de educación universitaria. Revista Multidisciplinaria Saber Universitario, (3), 18-25. Recuperado de https://saber755.webnode.com.ve/_f iles/200000012-d44eed44f0/Saber\%20Universitario\%203\%20-\%202020-4.pdf

Touron, R. y Santiago, R. (2015). El modelo Flipped Learning y el desarrollo del talento en la escuela. Revista de Educación, (368), 196-208. doi:10.4438/1988-592X-RE-2015-368-288

Universidad Pedagógica Experimental Libertador. (2006). Manual de trabajos de grado de especialización y maestrías y tesis doctorales. Caracas: Fondo Editorial de Universidad Pedagógica Experimental Libertador. https://ctgis.we bnode.es/_files/200000009-28a2c299c8/Manual\%20Tesis\%20UPEL\%202006.pdf

Vasilevska, D., Rivza, B., Pivac, T., Alekneviciene, V. y Parlinska, A. (2017). Analysis of the Demand for Distance Education at Eastern and Central European Higher Education Institutions. Journal of Teacher Education for Sustainability, 19(1), 106-116. doi: 10.1515/jtes-2017-0007

Watts, C.y Lee, L. (2017). Las TIC como herramientas de inclusión educativa. Acta Scientiae Informaticae, 1(1), 92-97. Recuperado de https://bit.ly/2OTWasy

Zabala-Vargas, J. E., Zabala-Vargas, S. A., Lizcano-Dallos, A. R. y Lizcano-Reyes, R. N. (2017). La tecnología educativa como elemento de transformación docente, su calidad, pertinencia, cobertura e impacto en el modelo de educación 
José Mercedes Estrada, et al. Formación educativa en y desde las Tecnologías de Información y Comu...

en Colombia. Memorias CIMTEDXXII Congreso Internacional sobre Educación Bimodal, "Competencias Digitales, Innovación y Prospectiva”, p.p. 63-78. Medellín, Colombia. Recuperado de https://bit.ly/2G5kc2H

\section{INFORMACIÓN ADICIONAL}

Cómo citar: Estrada, J.M. y Bennasar García, M.I. (2021). Formación educativa en y desde las Tecnologías de Información y Comunicación (TIC) en educación secundaria: el reto de hoy. Revista Educación, 45(2). Recuperado de http://doi.org/10.15517/revedu.v45i1.43424 